\title{
An Evaluation Study of Various Excitation Signals for Electrical Impedance Spectroscopy
}

\author{
Mahdi Rajabzadeh ${ }^{1}$, Jonathan Ungethuem ${ }^{1}$, Holger Mandry ${ }^{1}$, Carolin Schilpp ${ }^{2}$, Oliver Wittekindt ${ }^{2}$, Maurits Ortmanns ${ }^{1}$ \\ Institute of Microelectronics ${ }^{1}$, Institute of General Physiology ${ }^{2}$, University of Ulm, Ulm, Germany. \\ Email: Mahdi.rajabzadeh@uni-ulm.de
}

\begin{abstract}
Electrical Impedance Spectroscopy (EIS) is a popular method for investigating tissue properties. Implementing the signal generator for EIS measurements with a suitable excitation signal type is thereby one of the two system components. The choice of the excitation signal defines the measurement speed, signal-to-noise ratio (SNR), total area and power consumption of the system, and many more properties. Signal types such as analog single-tone, analog multi-tone, linear feedback shift registers (LFSR), and single-tone Sigma Delta Modulated $(\Sigma \Delta M)$ are proposed in the state of the art. In this work an EIS setup is implemented and successfully tested with all the mentioned signal types to evaluate their properties on impedance models as well as in vitro cell layers. It is proposed to combine the $\Sigma \Delta M$ with the multi-tone excitation signal yielding a very versatile excitation generator. Multi-tone $\Sigma \Delta \mathrm{M}$ are as fast as analog multitones, while benefiting from a binary output and thus less system complexity. The implemented EIS setup is used to perform EIS measurement for biological samples. The results show a very good matching with the reference for all the excitation signals.
\end{abstract}

\section{INTRODUCTION}

Tight junctions connect adjacent epithelial cells in the lung. Their functionalities allow them to facilitate passive transport of Chloride ions [1]. One method to investigate the influence of different parameters on the permeability and the transfer processes across the tight junctions is electrical impedance spectroscopy (EIS). EIS measurements reveal information on the transepithelial electrical resistance (TEER) and electrical capacitance. These parameters indicate the barrier properties of the cells grown on permeable membranes [1].

In order to characterize the sample under test (SUT), within the EIS setup a signal generator excites the SUT and then its response is recorded (Fig.1). Recently, the state of the art (SoA) focused on the implementation of various excitation signal generators [2],[3]. Signal generators in a potentiostatic measurement need to generate excitation signals in the voltage domain and thereby the response is in the current domain. In contrast, in galvanic measurements [4], SUT is excited with current sources and the response is recorded in the voltage domain. Excitation signals can have different forms such as single-tone frequency [5], multi-tone [6], pseudo random noise [5], and sigma-delta modulated [7]. These signals can be divided to two main categorizes of analog (multi-valued) and digital (binary) excitation signals. Analog excitation signal sources [6], [5] are realized by use of a digital to analog converter (DAC). The realization of these signal sources for multi-electrode arrays becomes a bottleneck, since the implementation of several signal sources could consume large onchip power and area. On the other hand, digital signal sources can be realized by use of global bitstream modualtion, such as a sigma delta modulator $(\Sigma \Delta \mathrm{M})$ DAC or linear feedback shift register (LFSR), and only two local switches

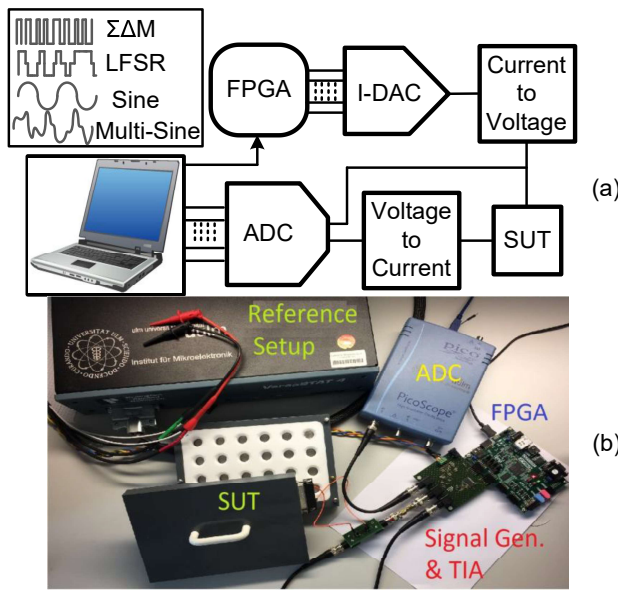

Fig. 1. a) Overview of a potentiostatic EIS setup with exemplary excitation signals. b) Implemented EIS setup and the utilized reference system.

at the multi-electrode array can perform the acutal 1-bit D/A conversion. [7], [5]. Due to digital characteristics of the signals, they are immune from noise and they can be easily distributed and multiplexed between multi-electrode arrays. Several digital signal sources can be implemented globally where area and power consumption is of lower concern.

Excitation signals for EIS can be classified based on the signal content to single-tone, multi-tone or wideband noise like signals. Single-tone excitation signals contain one frequency signal which is swept in the frequency range of interest [5]. The main drawback of this technique is its long evaluation time since several frequencies must be swept one by one [5]. On the other hand, multi-tone signals contain several frequencies and the SUT is simultaneously excitated by all the used frequencies. In this manner the evaluation time is reduced, however due to the addition of several frequencies, the summed amplitude becomes larger and more care has to be taken to not harm the SUT. Therefore, the amplitude of each individual frequency must be reduced, which leads to smaller signal to noise ratio (SNR). Noise like excitation tries to cover the overall frequency range at once, but a similar reasoning as for multi-tone excitation can be done concerning the maximum amplitude and SNR.

This work presents a board level EIS setup for the evaluation of various potentiostatic excitation waveforms with the goal to evaluate and compare their advantages and disadvantages concerning applicability and potential future chip integration. It is proposed to combine the multi-tone with $\Sigma \Delta \mathrm{M}$ digital excitation. The proposed method is tested to investigate the electrical properties of in vitro epithelial cell cultures. The results are verified by reference setup using a commercial EIS. 


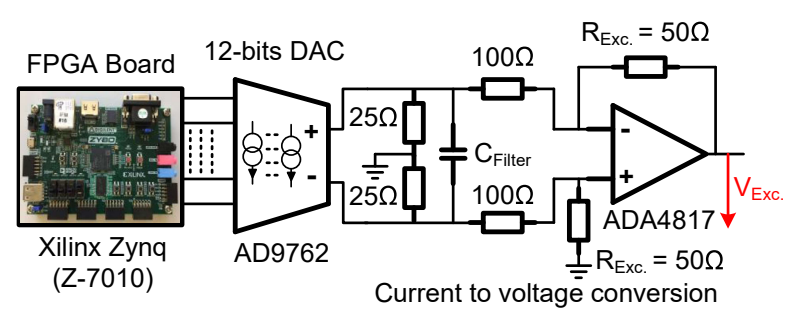

Fig. 2. Proposed voltage signal generator architecture.

\section{SYSTEM ARCHITECTURE}

The proposed EIS setup, shown in Fig. 1 consists of a signal generator, a sample under test (SUT), and a transimpedance amplifier (TIA) as current sensor, which have been implemented on a 2-layer Printed Circuit Board (PCB). Each part of the EIS setup is described in detail in the following.

\section{A. Signal Generator}

Potentiostatic measurements are in need of a voltage excitation source, which is typically been realized by a digital to analog converter (DAC) [6]. In this work, the signal generator is implemented by a current-output DAC followed by a current to voltage (C/V) converter (Fig.2). This architecture is chosen for two reasons. First, excitation voltages are typically in the range of few tens of $\mathrm{mV}$ for safety and biocompatibiltiy reasons [7]. Thus, utilizing a voltage-output DAC would mean only few least significant bits (LSB) of the DAC would be used to generate the signal, which translates to a small signal to noise ratio (SNR). In the proposed implementation the DAC uses the full bit-width to generate the output signal in the current domain (with higher signal content) and then it is converted to the voltage domain, where the DAC signal and noise are equally scaled. Second, in a future experiment it is intended to implement a galvanic measurement on the same board. Therefore, the current output DAC can then be reused to directly excite the SUT in current domain.

A field-programmable gate array (FPGA) is used to generate the bit stream for the used DAC. As shown in Fig.2, the FPGA board (Xilinx Zynq ${ }^{T M}$ Z-7010) provides a 12-bit output to the DAC (AD9762). The AD9762 has a nominal full-scale output current of $2 \mathrm{~mA}$ to $20 \mathrm{~mA}$ and $>100 \mathrm{k} \Omega$ output impedance. It can support update rates up to $125 \mathrm{MSPS}$, which makes it suitable for generating fast excitation signals. The C/Vconverter is realized with an ADA4817 amplifier, which is unity-gain stable featuring high speed $\left(f_{3 \mathrm{~dB}}=1050 \mathrm{MHz}\right)$, and extremely low output impedance (less than $0.1 \Omega$ ). This amplifier can be used for very fast voltage excitation signals and can drive a very low impedance load. In order to assure the safety of the SUT with nominal full-scale output current of $2 \mathrm{~mA}, R_{E x c .}$. (Fig.2) is fixed to $50 \Omega$. This leads to a maximum excitation voltage of $V_{\text {Exc. }}= \pm 20 \mathrm{mV}$.

\section{B. Sample Under Test}

Fig.3.a shows the structure of an epithelial cells that are connected to each other via the tight junctions [1].Transepithelial electric resistance (TEER) is modeled by a resistor and $C_{\mathrm{CL}}$ models the cell layer capacitance. The electrode surface can be modeled as capacitance $C_{\mathrm{DL}}$, which is also known as double layer capacitance and $R_{\mathrm{S}}$ represents the solution resistance [1]. Performing an EIS measurement on a SUT without a cell leads to an impedance characteristic as shown in Fig.3.b

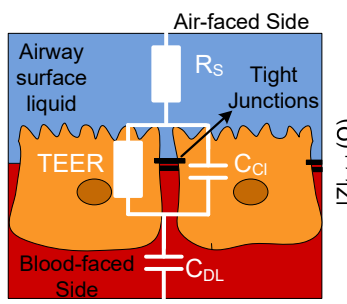

a)

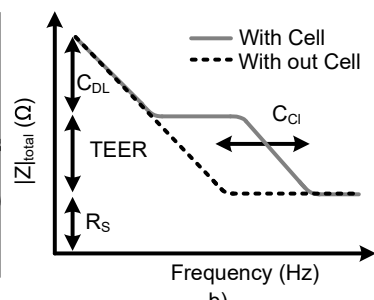

b)
Fig. 3. a) Epithelial cell and its electric model, b) Electric response of an Epithelial cell.

dashed line. At low frequency the electrode capacitance $\left(C_{\mathrm{DL}}\right)$ is dominating the total impedance. Increasing the frequency leads to a plateau proportional to the solution resistance $R_{\mathrm{S}}$. Adding the cells to the SUT will change the impedance characteristic as shown in Fig.3.b where at moderate frequencies the impedance becomes a plateau proportional to TEER. At higher frequencies $C_{\mathrm{CL}}$, which is parallel with TEER, starts to dominate the impedance and thereby, decreases the impedance. Additional information can be extracted from the phase change, which was not implemented in the first version of the evaluation routine, but is an easy extension of the system for future work.

\section{Transimpedance Amplifier}

The current recorder of the potentiostatic setup is simply implemented by a resistive transimpedance amplifier (R-TIA) followed by an analog to digital converter as shown in Fig.4. In this work an external ADC (PicoScope 4262) with a resolution of 16 bits at $10 \mathrm{MS} / \mathrm{s}$ is used for fast prototyping. The input parasitic capacitance by cables and circuitry is modeled by $C_{\mathrm{P}}$ at its input node, which varies due to the length of the cable and the electrode array from $10 \mathrm{pF}$ to $200 \mathrm{pF}$. In order to stabilize the R-TIA, $C_{\mathrm{F}}$ is introduced in parallel to the transimpedance resistor $R_{\mathrm{FB}}$. The main key components in the R-TIA are the amplifier and the value of the feedback resistor $\left(R_{\mathrm{FB}}\right)$ [8]. As reported in [8], the maximum achievable frequency of an R-TIA is inversely proportional to its transimpedance $\left(R_{\mathrm{FB}}\right)$ and the gain bandwidth (GBW) of the amplifier. Therefore, in order to achieve high transimpedance with high bandwidth, the amplifier ADA4817 has been chosen for the TIA. This amplifier has not only an ultrahigh speed $\left(f_{3 \mathrm{~dB}}=1050 \mathrm{MHz}\right)$, but also has a very good noise performance of $4 \mathrm{nV} / \sqrt{\mathrm{Hz}}$ and a noise corner frequency of $\approx 5 \mathrm{kHz}$. The R-TIA is designed based on [8] and was successfully tested for several $R_{\mathrm{FB}}=$ $10 \mathrm{M} \Omega, 1 \mathrm{M} \Omega$, and $5 \mathrm{k} \Omega$ on different PCBs.

Those different values of $R_{\mathrm{FB}}$ allow the design to detect low input currents due to an integrated noise of only $\approx 200 \mathrm{pA}$ over $B W=1 \mathrm{~Hz}-100 \mathrm{kHz}$ using the highest transimpedance, and also allow quite high currents $\approx 0.3 \mathrm{~mA}$ (saturation of the TIA using the lowest transimpedance). By using a commercial reference measurement setup (VersaStat 4), it was found for the available electrode setup (Fig.1) together with the used Phosphate buffered saline (PBS) solution that the maximum and minimum expected impedance can expanse from $\approx 50 \Omega$ to $\approx 500 \mathrm{k} \Omega$, respectively, and the interesting frequency range was given to be from $1 \mathrm{~Hz}$ to $100 \mathrm{kHz}$ by the collaborating physiologists. Despite the large dynamic range and frequency range of the utilized system, for the further measurements within the paper $R_{\mathrm{FB}}$ was fixed to $5 \mathrm{k} \Omega$, while the frequency range was fixed to $1 \mathrm{~Hz}-100 \mathrm{kHz}$. 


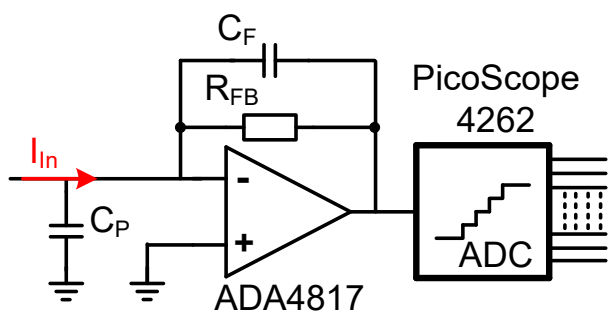

Fig. 4. The utilized current recording and digitization system.

\section{TyPES OF EXCITATION SIGNALS}

A distinguishing part of an EIS setup is the type of excitation signal, since it determines various parameters such as SNR, excitation time (speed), maximum excitation peak amplitude, etc. In this section various signal types are discussed. Then, by utilizing the available EIS system, all signal types are used to perform EIS measurements and the results are compared.

\section{A. Analog Single-tone Excitation Signal}

In case of the commonly used analog single-tone frequency excitation, the SUT is being excited by a fixed amplitude analog sinusoidal, whose frequency varies in the range of interest. In order to have a linear response of the electrode and SUT, the sinusoidal amplitude is limited to small excitation voltages of below $\pm 10 \mathrm{mV}$. In this method, the response of the system can be simply derived and digitized, but it can also be multiplied by sinusoidal and cosinusoidal signals of the same frequency to extract real and imaginary parts of the impedance of the SUT. This is is also known as phase sensitive analog lock-in detection [5]. Alternatively, digital lock-in detection can be performed which eliminates the limitations of the analog lock-in detection [7]. In this method the response of the SUT is digitized and then a fast Fourier transform (FFT) is applied. Since the system shows a linear behavior the power of the SUT response on the known frequency bin can be extracted and compared with the original power of the excitation signal bin. In this way the relation between these two powers indicate the properties of the SUT. Digital lockin detection is performed on all of the excitation methods investigated in this paper in order to improve the SNR.

A $\pm 10 \mathrm{mV}$ analog sinusoidal with frequency range from $1 \mathrm{~Hz}$ to $100 \mathrm{kHz}$, distributed semi-logarithmically over five decades, is used to perform the EIS measurements. In each decade, 9 tones are distributed linearly, which leads to a total number of 45 tones in the total frequency range. Fig. 5 shows the power spectral density of one of the utilized single-tone excitation signals. The biggest drawback of this technique is its very slow measurement time, since the system is sequentially excited with each individual frequency.

\section{B. Analog Multi-tone Excitation Signal}

In order to speed up analog single-tone measurements, analog multi-tone measurements were proposed [9], [6], where several frequencies are added to simultaneously excite the SUT. The purpose was to speed up the measurements of the single-tone excitation. The main drawback of this addition is that the maximum amplitude of the summed tones will become bigger than the individual tone. By shifting the sinusoidal signal in time - or in another words - by adding a suitable phase shift to each frequency, this drawback can be alleviated [9]. The crest factor (CF) shows the efficacy of this suppression: the $\mathrm{CR}$ is
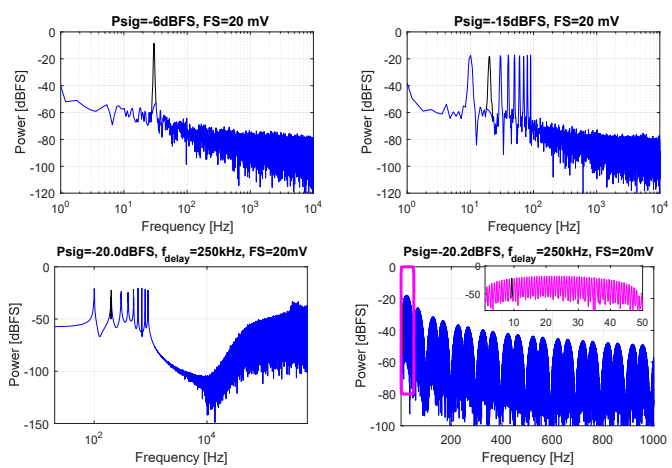

Fig. 5. Measured power spectral density (PSD) of the utilized excitation signals: analog single-tone (up-left), analog multi-tone (up-right), multi-tone $\Sigma \Delta \mathrm{M}$ (down-left), and LFSR (down-right).

defined by the ratio of the signal's peak value to its root mean square (RMS) value [9]. In order to have a high resolution in each decade, using the introduced method in [9], 9 tones, which are distributed linearly in each decade (same as in the single-tone method), are added. The resulting signal has a $\mathrm{CF}$ $\approx 2$. In order to not exceed a maximum excitation amplitude of $\pm 20 \mathrm{mV}$ (as maximum swing of the signal generator) with the resulting $\mathrm{CF}$, each individual frequency must have an amplitude of $\approx \pm 3.6 \mathrm{mV}$. EIS measurements are done for five decades in sequential manner form the first decade $1-10 \mathrm{~Hz}$ to the last decade $10-100 \mathrm{kHz}$, where Fig. 5 shows the frequency domain of the used excitation signal in the second decade.

\section{Sigma Delta Modulator Excitation Signal}

Digital signal excitation is a very attractive choice due to their higher immunity to noise, easy multiplexing, easy implementation of the 2 reference voltages, etc. [7]. A sigmadelta modulator $(\Sigma \Delta \mathrm{M})$ DAC consists of two parts, a digital modulator and an analog low-pass filter, which removes the high frequency quantization noise. As it was proposed in [7], the analog low-pass filter can be removed and the 1-bit digital output of the modulator can directly excite the SUT as a binary analog signal, which results in a very small area of the excitation signal source. However, in order to ensure safe operation, the specifications of the used $\Sigma \Delta M$ and its digital output levels must be carefully chosen. The reported $\Sigma \Delta \mathrm{M}$ used a single-tone frequency excitation, which resulted in a slow EIS similar as for single-tone measurements [7]. In this work, it is proposed to combine the multi-tone excitation signal with the $\Sigma \Delta \mathrm{M}$ approach, which will then speed up the EIS measurement similar as in the multi-tone approach, but with the easier binary implementation.

The modulator is implemented as third order feed-forward (CIFF) architecture. The sampling frequency is fixed to $f_{\mathrm{s}}=$ $20 \mathrm{MHz}$ and the binary output levels are $V_{\mathrm{p}, \mathrm{n}}= \pm 20 \mathrm{mV}$, which ensure safety of the excitation signal for the SUT [7]. The digital modulator is optimized for its required bit-widths in each stage to reduce the required hardware. Since the analog low-pass filter is omitted, it does not make sense to specify an oversampling ratio (OSR). The modulator is designed for 12 bits input and it utilizes a 12 bits digital integrator in its first stage followed by a 10 bits and 8 bits digital integrator at the second and third stages, respectively. The resulting modulator is implemented on the FPGA. An output spectrum of the synthesized modulator for a multi-tone excitation signal is shown in Fig.5. One draw back of a $\Sigma \Delta \mathrm{M}$ is its limited 
TABLE I. COMPARISON BETWEEN THE DIFFERENT EXCITATION SIGNAL TYPES.

\begin{tabular}{|c|c|c|c|c|}
\hline Method & $\begin{array}{c}\text { Spectrum } \\
\text { Engineering }\end{array}$ & Speed & SNR & $\begin{array}{c}\text { Binary/ } \\
\text { Multi-valued }\end{array}$ \\
\hline $\begin{array}{c}\text { Single-tone } \\
\text { Sweep }\end{array}$ & $\mathbf{+}$ & - & $\mathbf{+}$ & Multi-valued \\
\hline $\begin{array}{c}\text { Analog } \\
\text { Muli-tone }\end{array}$ & $\mathbf{+}$ & $\mathbf{+}$ & $\mathbf{+}$ & Multi-valued \\
\hline $\begin{array}{c}\text { LFSR } \\
\Sigma \Delta M\end{array}$ & $\mathbf{+}$ & $\mathbf{+}$ & Binary \\
\hline $\begin{array}{c}\Sigma \Delta \text { Ming-tone } \\
\Sigma \Delta M \\
\text { Multi-tone }\end{array}$ & $\mathbf{+}$ & $\mathbf{+}$ & $\mathbf{+}$ & Binary \\
\hline
\end{tabular}

maximum stable input amplitude (MSA), which is $\approx-6 \mathrm{dBFS}$ for the implemented modulator. For the multi-tone $\Sigma \Delta \mathrm{M}$, the excitation signals employ the same 9 linearly distributed tones as the analog multi-tone are used. Since the maximum input cannot exceed $-6 \mathrm{dBFS}$, each tone's amplitude must be $\pm 2 \mathrm{mV}$. This results to a smaller SNR for the $\Sigma \Delta \mathrm{M}$ excitation signal in comparison to the multi-level, analog multi-tone.

\section{Linear-Feedback Shift Register Excitation Signal}

One of the applications of linear-feedback shift registers (LFSR) is generating pseudo-random noise [5]. LFSRs are easy to build, and usually are made of few delay elements and digital EXOR blocks. This property and their digital output levels make them very attractive for a wide-band signal generator. However, engineering their spectrum is not as easy. As an example, in [5] it is proposed to combine the output of few delay element to achieve a concavely shaped spectrum with the penalty of increasing the complexity of the system and the output digital levels to three states $(+1,0,-1)$. Still, this concave shape leads to small amplitudes for small frequencies, where the SUT has usually its largest impedance (Fig.5) [5]. With this configuration for the lower frequencies, the resulting excitation current becomes small at low frequencies, or - in another words - the SNR becomes small. This leads to an inaccurate response for the interesting frequencies. Another part, which needs to be engineered, is the location of the tones and their distance to each other. In [5], the first tone was placed at $\approx 800 \mathrm{~Hz}$ and then 63 tones are distributed with linear steps of $\approx 800 \mathrm{~Hz}$ to $\approx 50 \mathrm{kHz}$. In the presented study, the interesting frequency range is form $1 \mathrm{~Hz}$ to $100 \mathrm{kHz}$. Therefore, the same structure as in [5] has been chosen but with three different delay frequencies $\left(f_{\text {delay }}\right)$ in order to generate the required spectra. Fig.5 shows the used LFSR spectrum in the frequency range of $\approx 0.9 \mathrm{~Hz}$ to $\approx 50 \mathrm{~Hz}$.

\section{Measurement Results And Discussion}

Fig.6 shows the results of the proposed excitation sources within the prototyped EIS system tested on an RC high-pass filter for all different signal types. All methods show a very good matching with the reference curve measured with the VersaStat 4 (Princeton Applied Research). Tab.I summarizes the pros and cons of the utilized signal types. The $\Sigma \Delta \mathrm{M}$ excitation signal with the multi-tone input is as fast as the analog multi-tone excitation signal but with the additional benefit of having binary output levels. These properties make the $\Sigma \Delta \mathrm{M}$ very practical to be implemented on an integrated circuit as it results a very compact signal generator. However, due to their limited input range, their SNR is slightly smaller than that of the multilevel, analog multi-tone. This issue could be alleviated by using $\Sigma \Delta \mathrm{M}$ with a higher input range. As highlighted in Fig.6, the LSFR shows a deviation from the

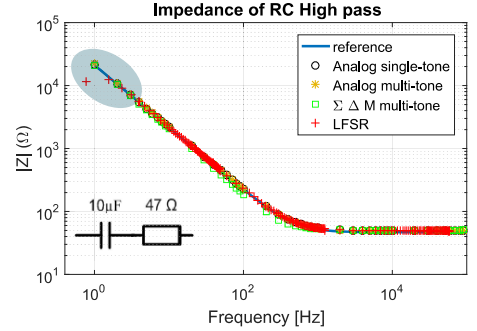

Fig. 6. Measured impedance of RC high pass with all the excitation signal types.

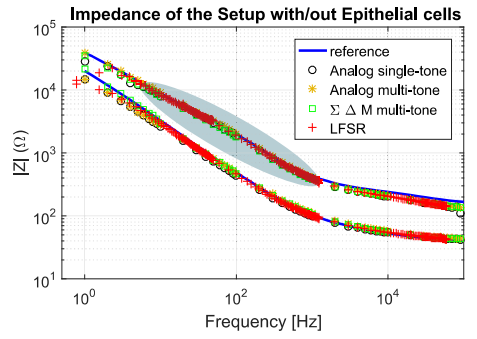

Fig. 7. Measured impedance of the electrode setup without Epithelial cells (down curve) and with Epithelial cells (up curve).

ideal curve around $1 \mathrm{~Hz}$, which is due to its small excitation signal at those frequencies.

The EIS system was also utilized to measure an in-vitro cultured human bronchial epithelial cells to prove the functionality of the prototype system for real biomedical scenarios. The cells were cultivated on a transwell filter with a $0.33 \mathrm{~cm}^{2}$ surface and cultivation time was for 28 days and up. First measurements were done with only the PBS solution within the used CellZScope insert holder [10] and without the cells nor the transwell filter inserts (lower curve in Fig.7). The result is the expected behavior of just the electrodes in PBS solution. Introducing the epithelial cells which are mounted on the transwell filter into the CellZScope insert holder, changes the overall shape of the filter (upper curve in Fig.7). The impedance is increased over the complete frequency range, but especially in the frequency range from about $20 \mathrm{~Hz}-$ $120 \mathrm{~Hz}$ the typical shape of a transepithelial layer is seen, with a slight bump in the rolloff, as expected from Fig.3. Fitting the resulting curve with the original model in Fig.3, a TEER $\approx 100 \Omega$ can be derived. The same setup was again used with the reference measurement (Versatat 4) as well as with the various signal sources, which proves the excellent matching using our prototype system and all its signal types.

\section{CONCLUSION}

In this paper, an electrical impedance measurement system with different signal excitation types has been presented. The utilized setup has been tested with different excitation signals in order to show the functionality of each method and it is successfully used to measure the impedance spectra of epithelial cells. It is concluded that a digital $\Sigma \Delta \mathrm{M}$ with multitone excitation signals is more beneficial than analog singletone, multi-tone sweep, and linear feedback shift registers (LFSR), due to its binary output and easy spectral engineering.

\section{ACKNOWLEDGMENT}

The authors would like to thank Dipl-Ing Andreas Herkle with the help in FPGA programming. This work is funded by DFG GRK 2203 (PULMOSENS). 


\section{REFERENCES}

[1] Benson, K., et al. "Impedance-based cell monitoring: barrier properties and beyond." Fluids and Barriers of the CNS 10.1 (2013): 5.

[2] Onet, Raul, Ana Rusu, and Saul Rodriguez. "High-Purity and WideRange Signal Generator for Bioimpedance Spectroscopy.” IEEE Transactions on Circuits and Systems II: Express Briefs (2018).

[3] Kassanos, Panagiotis, and Iasonas F. Triantis. "A CMOS multi-sine signal generator for multi-frequency bioimpedance measurements." Circuits and Systems (ISCAS), 2014 IEEE International Symposium on. IEEE, 2014.

[4] Allegri, Daniele, et al. "CMOS-Based Multifrequency Impedance Analyzer for Biomedical Applications.” Circuits and Systems (ISCAS), 2018 IEEE International Symposium on. IEEE, 2018.

[5] Ivanisevic, Nikola, et al. "Impedance spectroscopy systems: Review and an all-digital adaptive IIR filtering approach." Biomedical Circuits and Systems Conference (BioCAS), 2017 IEEE. IEEE, 2017.

[6] Yang, Lang, and Tom Chen. "A compact signal generation and acquisition circuit for electrochemical impedance spectroscopy." Biomedical Circuits and Systems Conference (BioCAS), 2016 IEEE. IEEE, 2016.

[7] Rajabzadeh, Mahdi, et al. "Evaluation of single-bit sigma-delta modulator DAC for electrical impedance spectroscopy." Biomedical Circuits and Systems Conference (BioCAS), 2017 IEEE. IEEE, 2017.

[8] Rajabzadeh, Mahdi, et al. "Comparison Study of Integrated Potentiostats: Resistive-TIA, Capacitive-TIA, CT $\Sigma \Delta$ Modulator.” Circuits and Systems (ISCAS), 2018 IEEE International Symposium on. IEEE, 2018.

[9] Yang, Yuxiang, et al. "An improved crest factor minimization algorithm to synthesize multisines with arbitrary spectrum." Physiological measurement 36.5 (2015): 895.

[10] https://www.nanoanalytics.com/de/produkte/uebersicht.html 